\title{
Predictors for the Choledocholithiasis. Retrospective Study for Bulgarian Patients
}

\author{
Stoyanka Dineva ${ }^{1}$, Krasimira Prodanova $^{2}$, Plamen Getsov ${ }^{3}$, \\ Borislav Vladimirov ${ }^{4}$ \\ ${ }^{I}$ Department Of Radiology, Medical Institute Of Ministry Of Interior - Sofia, Bulgaria \\ ${ }^{2}$ Technical University, Sofia, Bulgaria \\ ${ }^{3}$ Department Of Radiology, University Hospital "Queen Joanna " Sofia, Bulgaria \\ ${ }^{4}$ Clinical Center Of Gastroenterology At University Hospital " Queen Joanna “ Sofia, Bulgaria
}

\begin{abstract}
The presence of a periampullary duodenal diverticulum (PDD) is often observed during upper digestive tract barium meal studies and endoscopic retrograde cholangiopancreatography (ERCP). The impact of periampullary duodenal diverticulum for the formation of gallstones in the bile duct (choledocholithiasis) is widely accepted. In this study it using logistic regression modeling the appearance of choledocholithiasis depending periampullary duodenal diverticulum, to confirm the connection between them. The resulting model allows evaluation of the estimated probability of occurrence of this complication. The model is based on the 1719 recording of endoscopic retrograde cholangiopancreatography performed in the Department of Gastroenterology of the University Hospital "Queen Joanna", Sofia.
\end{abstract}

Keywords: periampullary duodenal diverticulum, choledocholithiasis, logistic regression

\section{Introduction}

The juxtapapillary duodenal diverticulum (JPDD) was first described by Chomel in 1710 [1]. The inflation of a diverticulum can cause compression of the ductus holedochus, which may result in an development of choledocholithiasis [2,3]. A few papers reported that the diverticulum had something to do with the incidence of choledocholithiasis. The aim of this study is to investigate if the presence of duodenal diverticula predisposes to the development of a choledocholithiasis.

A total 1719 patients who had undergone ERCP were studied retrospectively. They were divided into 2 groups-with and without periampullary duodenal diverticulum (PDD). Patients with a duodenal diverticula had a higher rate of choledocholithiasis. The duodenal diverticula is a risk factor for choledocholithiasis. A multiple logistic regression to obtain adjusted estimate of odds and to identify if a PDD is a predictor of choledocholithiasis, was performed. The software package STATISTICA 10.0 was used for analyzing the real data [16]. The impact of Periampullary duodenal diverticulum (PDD) for the formation of gallstones in the bile duct is widely accepted $[3,4,6,7]$. Scientific publications on these issues began to appear in the late 70 s with the introduction of endoscopic methods, in particular endoscopic retrograde cholangiopancreatography (ERCP).

The review of the literature reveals data on the incidence of choledocholithiasis in patients with PDD ranging from $12 \%$ to $68 \%[4,7]$. These theories put PDD in the role of an independent factor in the pathogenesis of choledocholithiasis. Applied by us logistic model, except that identify significant factors gives the possibility to calculate the probability that a certain amount of factors are expected to occur complication. Precisely because of the possibility to predict the probability of complication logistics model is applied in a variety of context, including cohort and case-control studies, problems in differential diagnosis and prediction and in the analysis of survey data [8-14].

\section{Material and metods}

A total of 1719 patients who had undergone ERCP for five year period were studied retrospectively. ERCP was performed in the Endoscopic department of the University

Hospital "Queen Joanna" in cooperation with the Department of Radiology. These patients were examined with attention to the localization of the papilla and the diverticula and to the relations between the localion and the choledocholithiasis.

The protocols include number of indicators like age, sex, pathology (before the examination) of bile duct, pancreas and etc., endoscopic findings, X-Ray findings, performed manipulations - sphincterotomy, lithotripsy, extraction, presence of duodenal diverticulum, laboratory data. The protocols were processed in a table with different indicators. In the present statistical analysis some of them are compared - age, sex, presence of duodenal diverticulum, attendant choledocholithiasis. 
Statistical model: Let assume that the categorical outcome of interest $Y$, is a dichotomous (binary) variable with categories $j=1,2$ and that $x=\left(1, x_{1}, x_{2}, \ldots, x_{s}\right)^{T}$ is a $(s+1)$ dimensional vector of covariates. The multiple logistic model specifies a conditional probability of the outcome given $x$ to be of the form:

$p_{j}(x)=P($ outcome falls in $j-$ th cathegory $/ x)=$

$$
=\frac{\exp \left(x \boldsymbol{\beta}_{j}\right)}{1+\exp \left(x \boldsymbol{\beta}_{j}\right)}, \quad j=1,2,
$$

$\boldsymbol{\beta}_{j}=\left(\beta_{j 0}, \beta_{j 1}, \ldots, \beta_{j s}\right), j=1,2$ are the regression parameters, $s$ is the number of independent variables in the model.

Let us consider $n$ observation for every component of $x$. Let us denote with $p_{i j}=p_{j}\left(x_{i}\right), i=1,2, \ldots, n ; j=1,2$ and for $Y_{j}\left(x_{i}\right)$ with $y_{i j}=1$ when the $j$-th category is chosen and 0 otherwise. The estimators of $\boldsymbol{\beta}_{j}, j=1,2$

are obtained by maximizing the likelihood for the observations $\left(Y_{i}, \mathbf{x}_{i}\right), i=1,2, \ldots, n$ based on the probabilities $p_{i j}$. The likelihood function is given in [15] by: $L=\prod_{i=1}^{n} p_{i 1}^{y_{i 1}} p_{i 2}^{y_{i 2}}$. The maximum likelihood estimator of the regression parameters $\boldsymbol{\beta}_{\mathbf{j}}=\left(\beta_{j 0}, \beta_{j 1}, \ldots, \beta_{j s}\right)$ is obtained from the system:

$\frac{\partial \ln L}{\partial \beta_{j k}}=\sum_{i=1}^{n}\left(y_{i j}-p_{i j}\right) x_{i k}=0, k=0, \ldots, s ; j=1,2$.

Newton-Raphson procedure is used to approximate the maximum likelihood estimator $\hat{\boldsymbol{\beta}}_{j}$. The coefficients $\hat{\boldsymbol{\beta}}_{j}$ were interpreted as estimates of logodds. A marginal one unit increase in $x_{j}$ brings an increase inlogoddsof the amount of $\hat{\boldsymbol{\beta}}_{j}$. For the dichotomous (binary) logistic regression model the likelihood function has the form:

$$
L=\prod_{i=1}^{n} p^{y_{i}}(1-p)^{1-y_{i}}
$$

where $y_{i}=0$ or 1 depending in which group the $i$-th observation belongs. The dichotomous models assumes that the logit function (logarithm of odds - log odds) is: $\ln \frac{p}{1-p}=\beta_{0}+\beta_{1} x_{1}+\ldots+\beta_{s} x_{s}$,

where $p$ is the probability that the outcome belongs to the group coded with " 1 ".

Testing of hypothesis concerning the regression parameters can include test of single parameter, test involving several parameters from the same regression, and joint tests involving parameters from different regressions. In multiple logistic regression, tests for contribution of one or more parameters from the same regression are usually constructed with a large sample Wald test, with test statistic

$$
Q_{w}=\boldsymbol{\beta}_{B}^{T}\left[\operatorname{Var}\left(\boldsymbol{\beta}_{B}\right)\right]^{-1} \boldsymbol{\beta}_{B},
$$

where $\operatorname{Var}\left(\boldsymbol{\beta}_{B}\right)$ is the estimated covariance sub-matrix for the corresponding parameters. If the null hypothesis is that the $q$-dimensional vector $\boldsymbol{\beta}_{B}=0$, then this statistic has approximately $\chi^{2}$ distribution with $q$ degrees of freedom validating these relationships. For contribution of a given parameter in the regression the following inequality for the probability of test statistics

$P\left(Q_{w} \geq \chi_{0,05 ; q}^{2}\right)<0,05$

must be satisfy, if the using p-level for significance is 0,05 .

\section{Results And Discussion}

A dichotomous logistic regression analysis was performed to identify the explanatory variables (factors) independently associated with choledocholithiasis appearance. The purpose of the constructed multiple dichotomous logistic model was to study the impact of the gender, age, PDD and cholelythiasis on the response variable - the presence or absence of choledocholithiasis.

Using Wald's test statistics and p-level for significance 0,05 we conclude that factors gender $(p=0,00004<0,05)$, age $(p=0,0042<0,05)$ and PDD $(p=0,000021<0,05)$ are independently associated with choledocholithiasis appearance (Tabl.1). Factor cholelythiasis is not predictive because $\mathrm{p}=0,198$ is greater than 0,05 . 
Table 1. The estimated parameters of the logistic regression model.

\begin{tabular}{|l|c|c|c|c|}
\hline Factor & gender & age & PDD & $\begin{array}{c}\text { cholely } \\
\text { thiasis }\end{array}$ \\
\hline $\begin{array}{l}\hat{\boldsymbol{\beta}}_{j} \\
\hat{\boldsymbol{\beta}}_{0}=-4,59\end{array}$ & $-0,323$ & 0,1006 & 0,5899 & $-0,093$ \\
\hline $\begin{array}{l}\text { Wald } \mathrm{p}- \\
\text { level }\end{array}$ & 0,00004 & 0,00001 & 0,000021 & 0,198 \\
\hline $\begin{array}{l}\text { Odds Ratio } \\
\text { (OR) }\end{array}$ & 0,71 & 1,106 & 1,8 & 0,91 \\
\hline $\begin{array}{l}\text {-95\%CL for } \\
\text { OR }\end{array}$ & 0,62 & 1,06 & 1,09 & 0,78 \\
\hline $\begin{array}{l}\text { +95\% CL for } \\
\text { OR }\end{array}$ & 0,81 & 1,15 & 2,98 & 1,05 \\
\hline
\end{tabular}

To illustrate the information in the Table 1 , let examine in details the relationship between choledocholithiasis and age. The fitted equation coefficient $\hat{\boldsymbol{\beta}}_{2}=0,006>0$ indicated that the higher levels of age, the greater the probability for choledocholithiasis presence (coded by 1 ). The value for estimated probability of choledocholithiasis presence in patients of age 12 years is

$$
P(12)=\frac{\exp (-4,59+0,1006 * 12)}{1+\exp (-4,59+0,1006 * 12)}=0,033 \text {, }
$$

and in patients of age 60 years is

$$
P(60)=\frac{\exp (-4,59+0,1006 * 60)}{1+\exp (-4,59+0,1006 * 60)}=0,806
$$

Distribution of all probabilities of the factor age estimated using constructed logistic model are presented in Fig.1 and for the factor PDD in Fig.2.

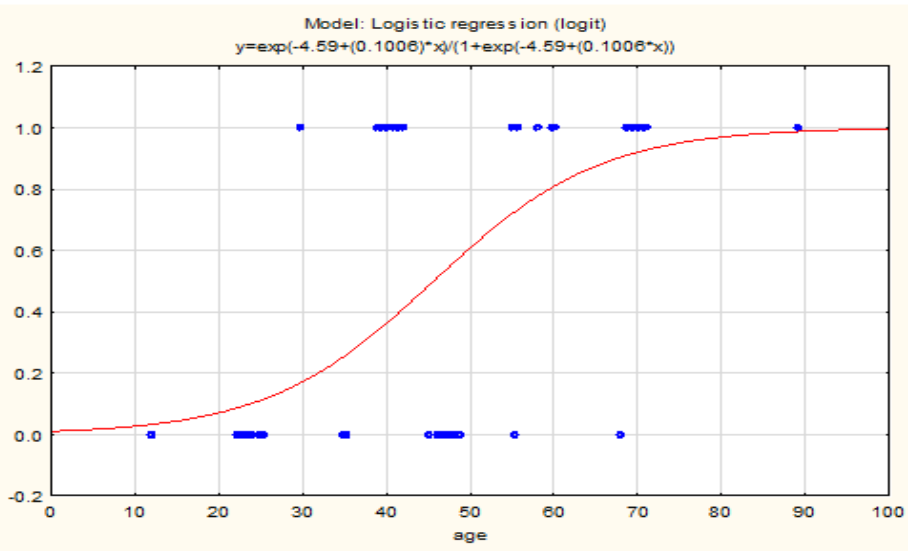

Fig.1: Probability distribution of the factor age estimated using logistic model. 


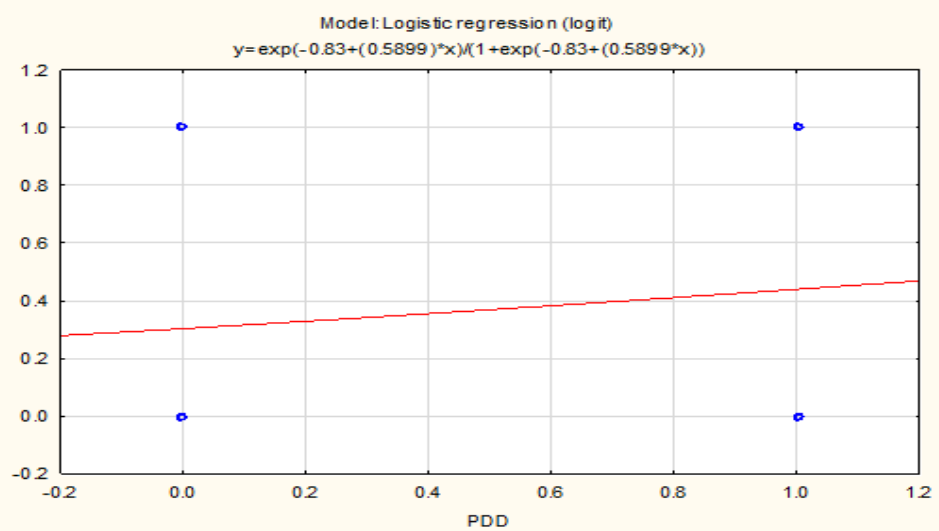

Fig.2: Probability distribution of PDD estimated using logistic model.

The fitted equation coefficient $\hat{\boldsymbol{\beta}}_{1}=-0,323<0$ indicated that the lower levels of gender (cods: 0 -male, 1 female) i.e. male, the greater the probability for choledocholithiasis manifestation.

\section{Conclusions}

From the foregoing it appears that billiopancreaticsystem and duodenum should be considered as a single system - changes in one component leads to changes in others. The etiology of complications of billiopancreatic system is complex. Although occurring at different frequencies, each of complications is good to explore both independently and as part of the range of pathology in this area. PDD is only part of this etiology. The study of dependence on it contributes to a possible assessment of risk of specific complications. The performed statistical analysis found that periampullary duodenal diverticulum is a predictor of complication choledocholithiasis.

\section{References}

[1]. ChomelJ.Duodenal Diverticula.Br. J. Radiol., 1933 ,№6,p: 577-587.

[2]. Emmanuel C, Konstantinos V, Konstantinos B, Konstantinos T, DimitriosB. Feasibility of Therapeutic Endoscopic RetrogradeэCholangiopancreatography for Bile Duct Stones inNonagenarians: a Single Unit Audit. J Gastrointestin Liver Dis. December, 2008 Vol.17, No 4, p.427-432

[3]. Chandy G, Hart WJ, Roberts-Thomson IC. An analysis of the relationship between bile duct stones and periampullary duodenal diverticula. J Gastroenterol Hepatol.,1997 Jan;№12(1), p:29-33.

[4]. Li ZH, Chen M, Liu JK, Ding J, Dong JH. Endoscopic shincterotomy in treatment of cholangiopancreatic diseases. World J Gastroenterol 2005;11 (17),p:2678-2680

[5]. Tan NC, Ibrahim S, Chen CM, Tay KH. Periampullary diverticulum causing biliary stricture and obstruction. Singapore Med J 2005;46, p:250-251.

[6]. Waters JA, Schmidt M, Pinchot JW, White P, Cummings OW, Pitt HA et al. CTvs MRCP: Optimal classification of IPMN Type and Extent. J GastroinestSurg (2008) 12, p:101-119

[7]. ZhangWZ, Xu MC, Shi ZH, Yang YL. Relationship between juxtapapillary duodenal diverticula and biliopancreatic diseases in patients over 60 years old. Di Yi Jun Yi Da Xue Xue Bao 2003; 23(9), p:952-3

[8]. K. Prodanova ,Pashkunova S., Modelling data for complications in diabetics using Logistic regression, Pliska Stud. Math. Bulgar., vol. 22, 2013, pp. 143-158.

[9]. S. Dineva, Prodanova K., Mlachkova D., Modeling Data for Pancreatitis in Presence of a Duodendal Diverticula using Logistic Regression,AIP Conference Proceedings, vol. 1570, 2014, pp. 39-44.

[10]. M. Negreva , Prodanova K., Vitlianova K., Alexandrova A., Prognostic Capacity of Oxidative Biomarkers in Paroxymal Atrial Fibrillation, IJBSAC, Vol. 1 (6), 2015, pp. 1-5.

[11]. M. Negreva , Georgiev S., Prodanova K., Vitlianova K., Arabadzhieva D., Interleukin-4 and Paroximal Atrial Fibrillation, Merit Res. J. of Med. and Med. Sci., Vol. 3 (7), 2015, pp. 271-277.

[12]. M. Negreva, Prodanova K., Georgiev S., Predictive Significance of Interleukin-6, Interleukin-8 and Tumor Necrosis Factor-Alpha in Paroximal Atrial Fibrillation, Sch. J. App. Med. Sci., Vol. 3 (8D), 2015, pp. 3013-3019.

[13]. M. Negreva, Georgiev S., Prodanova K., Significant Increase in C-Reaitive Protein and Serum Amyloid A in the Early Hours of Paroxymal Atrial Fibrillation, Cardiol Res, vol. 7 (1), 2016, pp. 1-8.

[14]. Y. Usunova., Prodanova K., Spasov L., Prediction of Early Postoperative Infections in Pediatric Liver Transplantation by Logistic Regression, AIP Conference Proceedings AMEE, 2016. (in press).

[15]. J. D. Jobson, Applied Multivariate Data Analysis, Vol. 2, Springer Verlag, 1991, ch. 8.

[16]. StatSoft, Inc., STATISTICA Manual (Data analysis software system), Version 10.0, 2010 